\title{
Muon track reconstruction and muon energy estimate in the KM3NeT/ARCA detector
}

\section{Agata Trovato $^{a}$, Evangelica Drakopoulou ${ }^{b}$ and Piera Sapienza ${ }^{* c}$ for the KM3NeT Collaboration}

a INFN-LNS, via S. Sofia 62, 95123 Catania, Italy

Email:atrovato@lns.infn.it

${ }^{b}$ N.C.S.R. Demokritos, Patriarchou Gregoriou and Neapoleos, Agia Paraskevi, Greece and National Technical University of Athens, Heroon Polytechniou 9, Zografou Campus, Greece

Email: liliadrakeinp.demokritos.gr

${ }^{c}$ INFN-LNS, via S. Sofia 62, 95123 Catania, Italy

Email:sapienza@lns.infn.it

\begin{abstract}
KM3NeT will be a network of deep-sea neutrino telescopes in the Mediterranean Sea. The ARCA detector to be installed at the CapoPassero site (Italy) is optimised for the detection of high-energy neutrinos of cosmic origin. The "golden channel" for neutrino astronomy with Cherenkov telescopes is the muon-neutrino charged-current interaction. The final-state muon has a track length in water (for neutrino energies above $1 \mathrm{TeV}$ ) of the order of kilometres and its trajectory is almost co-linear to the parent neutrino direction, thus pointing back to the neutrino source. The neutrino direction is reconstructed from the arrival times of the photons at the photomultiplier tubes. After an initial hit selection requiring space-time coincidences between hits, the reconstruction includes four consecutive fitting procedures. The median angular resolution reached is about $0.2^{\circ}$ for neutrino energies above $10 \mathrm{TeV}$. Together with the track reconstruction, also the muon energy has to be estimated. Since atmospheric neutrinos, that represent an unavoidable background, have a softer spectrum that the one expected for cosmic neutrinos, the reconstructed energy plays an important role in the optimisation of the detector sensitivity. The method described here to determine the muon energy is based on a Neural Network. The main aspects of the techniques employed for the track reconstruction and energy estimate are discussed and the results presented.
\end{abstract}

The 34th International Cosmic Ray Conference,

30 July- 6 August, 2015

The Hague, The Netherlands

\footnotetext{
*Speaker.
} 


\section{Introduction}

The main goal of neutrino telescope experiments is the observation of high energy neutrinos from cosmic sources. These instruments detect neutrinos indirectly through charged particles produced in weak neutrino interactions. In transparent media, relativistic particles can be detected through the light produced via the Cherenkov effect with a three-dimensional array of optical sensors. The charged-current muon-neutrino interaction represents the "golden channel" for high-energy neutrino astronomy with Cherenkov telescopes since the produced muons are highly penetrating particles. They reach track length of the order of kilometres for neutrino energies above $1 \mathrm{TeV}$. The flight direction of these particles is nearly collinear with the incident neutrino direction for neutrinos with energies above $10 \mathrm{TeV}$, thus the reconstruction of the muon direction makes pointing back to the neutrino cosmic source possible. A good angular resolution is of course crucial requisite for the search of cosmic neutrinos from point-like sources [1].

Events induced by neutrinos from astrophysical sources must be distinguished from $v_{\mu}$ and $\mu$ originating from the interaction of cosmic rays with the atmosphere. Atmospheric neutrinos are an unavoidable background but a key for a possible discrimination against cosmic neutrinos is their different spectrum, which is softer than the expected spectrum of cosmic neutrinos. Atmospheric muons can be rejected looking at upward-going events, which can be only produced by neutrinos, the only particles that can traverse the whole Earth without being absorbed. This rejection requires a reliable determination of the elevation angle, because downward-going atmospheric muon events outnumber upward-going muon events induced by atmospheric neutrinos by 5-6 orders of magnitude for typical neutrino telescope installation depths; hence even a small percentage of atmospheric muons mis-reconstructed as upgoing can represent a significant background.

$\mathrm{KM} 3 \mathrm{NeT}[3,4]$ is an international collaboration which aims to build a research infrastructure in the Mediterranean Sea hosting an underwater multi- $\mathrm{km}^{3}$ high-energy neutrino telescope. In the following a muon track reconstruction procedure and energy estimate appropriate for such a detector are described.

\section{The KM3NeT/ARCA detector}

The KM3NeT detectors will consist of three-dimensional arrays of 17 inch diameter pressureresistant glass spheres, the so-called DOMs (Digital Optical Modules), each equipped with 31 photomultipliers (PMTs) with 3-inch photocathode diameter and their readout electronics. For each PMT the start time and the duration of the signal above a predefined threshold (time over threshold, ToT) are recorded and sent to shore. The DOMs will be arranged in detection units, vertical strings anchored on the sea floor. The arrival time of single photons will be measured with nanosecond precision while the resolution on the PMT positions is expected to be about $10 \mathrm{~cm}$.

The detector array will be subdivided into building blocks of 115 detection units each. This modular design allows for a staged implementation and a installation in different sites. Two installation sites, one $40 \mathrm{~km}$ offshore Toulon in France at a depth of $2500 \mathrm{~m}$ and the other $80 \mathrm{~km}$ offshore Capo Passero in Italy at a depth of $3500 \mathrm{~m}$, are proposed to host the first detectors of the telescope. In response to the IceCube discovery of cosmic neutrinos [2] and the reported non-zero value for the neutrino mixing angle $\theta_{13}$, KM3NeT 2.0 will consist of two detectors with different granular- 
ity: KM3NeT/ARCA [4] at the KM3NeT-It site with two building blocks dedicated to high-energy neutrino astronomy and KM3NeT/ORCA [5], a single building block located at the KM3NeT-Fr site dedicated to the study of the neutrino mass hierarchy.

The reconstruction methods discussed in this paper have been developed for the KM3NeT/ARCA detector. A KM3NeT/ARCA building block consists of 115 detector units (DU), each containing 18 DOMs; the vertical distance between DOMs is $36 \mathrm{~m}$. The footprint is roughly circular with an average horizontal distance between adjacent DUs of $90 \mathrm{~m}$. Monte-Carlo simulations have been used to optimise the performance of the algorithms described in the following sections. A complete simulation is performed [6], including the neutrino interaction in the medium and the propagation of the resulting secondary particles [7], the light generation and propagation in water and the detector response in terms of PMT data sent to shore. In particular, atmospheric muons and muon bundles from cosmic ray air showers are generated with the MUPAGE event generator [8]. The simulation is based on the nominal detector geometry. Effects of position and orientation calibration uncertainties are expected to be small and are neglected at this stage. The depth and the optical water properties measured at the Capo Passero site (KM3Ne-It) have been used [9]. Background due to the presence of ${ }^{40} \mathrm{~K}$ in salt water has been simulated by adding an uncorrelated hit rate of $5 \mathrm{kHz}$ per PMT in a time window of $10 \mu$ s before and after the event. Moreover, the probability of two- three- and four-fold hit coincidences on a DOM due to the coincidences from ${ }^{40} \mathrm{~K}$ decays have been estimated by GEANT simulations and are included with a rate of 500,50 and $5 \mathrm{~Hz}$, respectively, per DOM. Both the single and coincidence rates are in reasonable agreement with the results from the prototype optical module deployed at the ANTARES site [10]. The effect of the bioluminescence light is negligible in the KM3NeT-It site (burst fraction about 1\% [11]).

\section{The muon track reconstruction}

The track reconstruction algorithm permits to estimate the muon direction, and consequently to infer the neutrino direction, using the information of the PMT spatial positions and the Cherenkov photon arrival times. The reconstruction code used in this work has been written to exploit the features of the multi-PMT DOM and is based on the reconstruction strategy described in [12] that was optimised for a detector using large area PMTs (8-10 inch). The new DOM developed by the KM3NeT collaboration has instead 31 small (3 inch) PMTs contained in a 17-inch glass sphere. It is thus necessary to substitute the charge information with the multiplicity of hits on the same DOM, to adapt the hit selection and to take into account the PMT directional sensitivity.

\subsection{Initial hit selection}

The presence of hits from background, mostly due to ${ }^{40} \mathrm{~K}$ decays in sea water, affects the reconstruction. The purity of the initial hit sample, defined as the ratio of the number of signal hits, Nhit $_{\text {sig }}$, over the total number of hits, Nhit $t_{\text {tot }}$, is shown in Fig. 1. A careful hit selection is therefore needed prior to the reconstruction procedure. The requirement of space-time coincidences between hits is a simple and efficient way to perform this task, since hits due to optical background are mostly uncorrelated. The code checks all hits for the different coincidence patterns: coincidences between PMTs of the same DOM, coincidences between hits on adjacent or next-to-adjacent DOM on the same string, coincidence between DOMs on nearby strings. Two hits occuring at times $\mathrm{t}_{1}$ and 

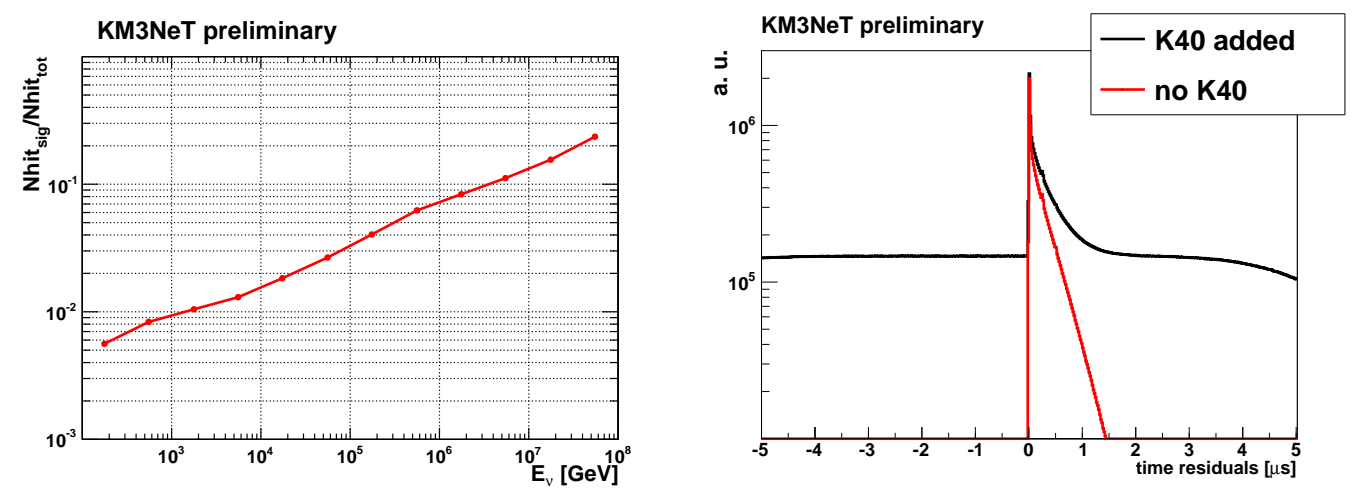

Figure 1: Left: Purity of the initial hit sample prior to reconstruction. Right: Time residuals distributions with respect to the simulated muon track (see sec. 3.2) for signal hits (red) and for events with the ${ }^{40} \mathrm{~K}$ contribution added (black).

$\mathrm{t}_{2}$ are considered coincident if $\Delta \mathrm{t}=\left|\mathrm{t}_{1}-\mathrm{t}_{2}\right|<5 \mathrm{~ns}$ if they are on the same DOM or if $\Delta \mathrm{t}<5 \mathrm{~ns}+\mathrm{t}_{\mathrm{d}}$ otherwise, where $t_{d}$ is the time required by the light to travel the distance $d$ between the two hits. Each coincidence pattern gives a "score" to the corresponding hit. These scores are calculated from the probability that the considered pattern can be randomly generated by background hits.

The hit with the highest score in the event is chosen as the "reference hit". A "causality filter" is then applied to reject background hits that are not in a causality relation with the reference hit. The causality filter is given by the relation:

$$
|\Delta t|-\frac{\Delta r}{v}<20 \mathrm{~ns} \quad \cap|| \Delta \mathrm{t}\left|-\frac{\Delta \mathrm{r}}{\mathrm{c}}\right|<500 \mathrm{~ns}
$$

where $\Delta t$ e $\Delta r$ are the time delay and space distance between each hit and the reference hit and $v$ is the group velocity of light in water.

The first term of 3.1 requires that the hit time delay $|\Delta t|$ is compatible (within a time window of $20 \mathrm{~ns}$ ) with the time taken by the Cherenkov wave front to travel the distance $\Delta r$. The second condition takes into account that, for large distances, light absorption does not allow the Cherenkov photon to reach PMTs far from the muon track. In this case, $\Delta t$ is compared with the time taken by the muon to travel the distance $\Delta r$. Hits that do not satisfy the causality filter are discarded.

\subsection{Fitting procedures}

After the initial hit selection the reconstruction proceeds through four consecutive fitting steps, each using the result of the previous one as starting point. Each fitting stage improves the accuracy of the reconstructed track direction.

The first step, called prefit, is a linear fit and therefore does not require an input track. At this stage, in order to obtain a linear relation between the hit positions and the track parameters, it is assumed that the hits occur on points that are located along the muon track.

The following fitting procedures are based on the maximum likelihood method and use a probability density function (PDF) for the time residuals, defined as $t_{\mathrm{r}}=t_{\exp }-t$ (see Fig. 1), where $t_{\text {exp }}$ is the expected time of arrival of the Cherenkov photon on the PMT and $t$ is the recorded hit time $t$. The $t_{\exp }$ can be calculated by simple geometrical considerations as a function of the 
track parameters assuming that the event starts at reference time $t_{0}$, the muon travels on a straight line with speed $c$, the Cherenkov light is emitted at a Cherenkov angle $\theta_{C} \approx 42^{\circ}$ with respect to the muon direction and the photons travel with a speed $c / n$, where $n$ is the refractive index of the medium. The PDF can be calculated fitting the time residual distribution extracted from the Monte Carlo simulations. However, in the likelihood fit that uses this PDF local maxima can appear due to the presence of large $t_{\mathrm{r}}$ for which the PDF is rather flat. For this reason, a robust estimator, called "M-estimator", is used after the prefit. The M-estimator likelihood has the form $-\sqrt{1+t_{\mathrm{r}}^{2}}$ and describes well the data for small time residuals while for large time residual it underestimates the probability, thus enhancing the possibility of finding the global maximum.

The third and fourth fitting procedures estimate the parameters of the track maximising likelihood functions extracted by Monte-Carlo simulations as described above. The PDF of the third fit is calculated not including background hits, while the one used in the fourth fit considers them.

Each of the fit procedure constrains the hit selection with respect to the track parameters resulting from the previous stage selecting only hits within a given orthogonal distance from the input track, in a time residual window and inside the PMT field of view. The hits part of four-fold coincidences are always selected and from the obtained list of hits only one hit per DOM is selected, the first in time among the ones with the highest score.

The efficiency of the algorithm is improved with an all sky scanning of all sky in steps of $3^{\circ}$ starting from the prefit track, thus generating 7200 tracks. These tracks are ordered on the basis of the number of hits, $\mathrm{N}_{\text {hit }}$, that fulfill the spatial and temporal relations described and the first 100 tracks with the higher $\mathrm{N}_{\text {hit }}$ are selected. For each direction the fitting procedures are performed and the solution with the highest likelihood per degree of freedom is chosen.

The algorithm described above yields an estimate of the muon position and direction. After this procedure an estimate of the interaction vertex and of the track length is added by back-projecting the hits onto the reconstructed track using the Cherenkov angle. At high energy the muon track is long enough for the neutrino interaction to occur often outside the instrumented volume; therefore a pseudo-vertex related to the first photon seen by the detector is estimated.

\subsection{Results}

The reconstruction output is the reconstructed track and an estimator of the fit quality, $\Lambda$, calculated as the maximum log-likelihood value per degree of freedom found in the fit. This variable is used to reject badly reconstructed events and in particular atmospheric muons misreconstructed as upgoing. In addition, the uncertainty on the track direction $\beta$ is estimated from the width of the likelihood maximum. The right plot in Fig. 2 shows the cumulative $\Lambda$ distributions of the atmospheric muons misreconstructed as upgoing and of upgoing atmospheric $v_{\mu}+\bar{v}_{\mu}$. The figure shows that a cut $\Lambda>-6$ will remove most of the atmospheric $\mu$.

The left plot in Fig. 2 shows the neutrino angular resolution, defined as the median of the angular distance between the simulated neutrino and the fitted track (black line). The light and dark blue bands indicate the $68 \%$ and the $90 \%$ quantiles, respectively. For reference also the median of the intrinsic angle, the angular distance between the simulated neutrino and the corresponding muon, is reported with a red line. A cut $\Lambda>-6$ is applied both for the calculation of the angular resolution and of the intrinsic angle. Below the $\mathrm{TeV}$ energy range the main contribution to the angular resolution is due to the intrinsic angle, while above $\sim 10 \mathrm{TeV}$ it reaches about $0.2^{\circ}$. 

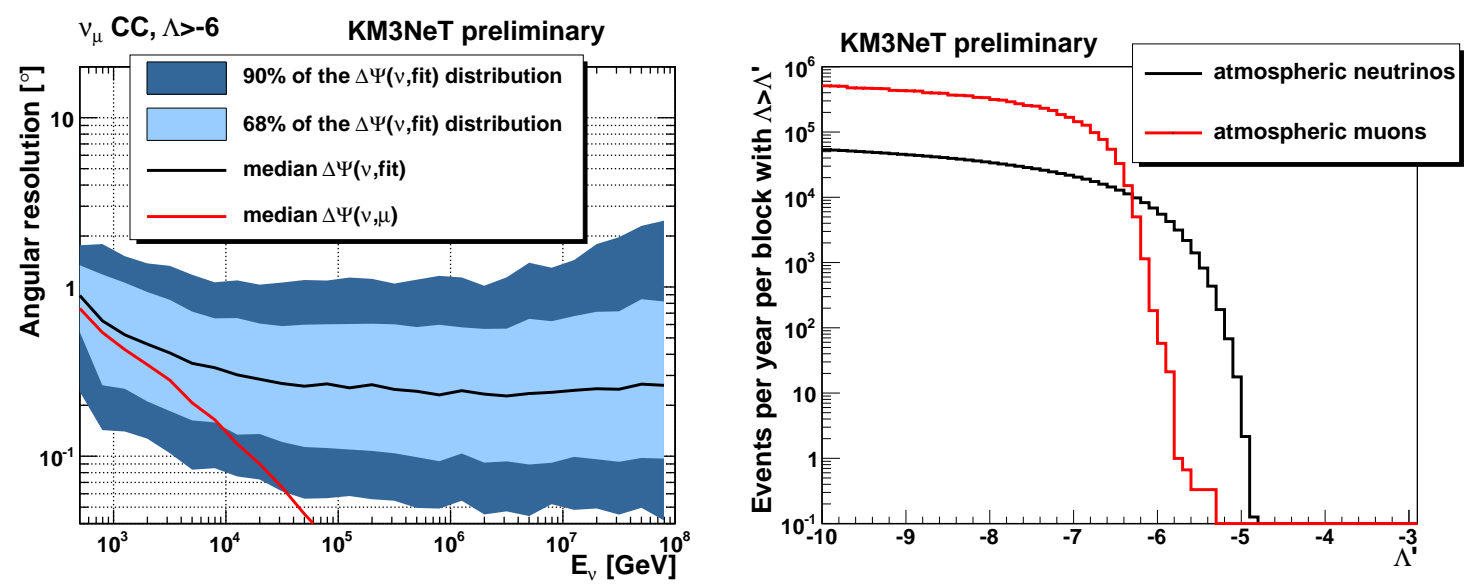

Figure 2: Left: Neutrino angular resolution estimate for muon neutrinos. Detailed description in the text. Right: Cumulative $\Lambda$ distribution of the atmospheric $\mu$ and atmospheric $v_{\mu}+\bar{v}_{\mu}$ reconstructed as upgoing.

To investigate the impact of ${ }^{40} \mathrm{~K}$, the reconstruction performance has also been studied without ${ }^{40} \mathrm{~K}$ hits. While the angular resolution is effectively unchanged, ${ }^{40} \mathrm{~K}$ background is found to reduce the number of reconstructed events by $20 \%$ at energies of a few $100 \mathrm{GeV}$ and by $5 \%$ at $E_{v}>1 \mathrm{TeV}$.

\section{Energy Reconstruction using a Neural Network}

High energy muons ( $\mathrm{E}>$ some $\mathrm{TeV}$ ) travel several kilometers in sea water and lose energy via ionisation and stochastic processes, such as bremsstrahlung, pair production, and photonuclear interactions. The total average energy loss of the muon is:

$$
-\frac{d E}{d x}=a(E)+b(E) E_{\mu}
$$

where $a \simeq 0.274 \mathrm{GeV} \mathrm{m}^{-1}$ accounts for the energy loss due to ionisation and $b \simeq 0.000349 \mathrm{~m}^{-1}$ is due to the stochastic energy loss. Muons with $E_{\mu}>1 \mathrm{TeV}$ lose energy stochastically, while for lower energies ionisation dominates. We describe a method to derive muon and consequently neutrino energy from the light collected when the $\mu$ passes through the instrumented detector volume.

Since the determination of the muon energy is based on the collection of light in PMTs, the energy reconstruction requires that muons have to travel an adequate distance inside the instrumented volume of the detector. Assuming a cylindrical shape of the detector, with height $\mathrm{h}$ and radius $\mathrm{R}$, the distance traveled inside the instrumented volume by well contained muons with reconstructed zenith angle $\theta_{\text {rec }}$ is estimated in the code as $\mathrm{L}=\mathrm{h} / 2+(\mathrm{R}-\mathrm{h} / 2) \sin \theta_{\text {rec }}$. For each event the distance $\mathrm{L}_{r e c}$ between the first and the last PMT position along the track is calculated and the event is accepted if $\mathrm{L}_{r e c}>0.3 \mathrm{~L}$ (containment selection).

The energy estimate is based on the employment of an artificial neural network, specifically a Multi-Layer Percepton (MLP) Neural Network, which is part of the TMVA package included in ROOT [13].

The quantities that have a strong dependence on the muon energy and are used as input variables to feed the Neural Network are:

i. The number of DOMs with hits used for the track direction reconstruction. 


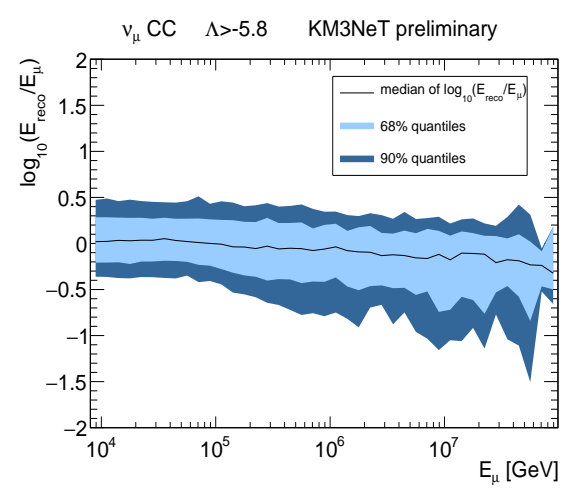

Figure 3: Median of $\log _{10}\left(E_{\mathrm{reco}} / E_{\mu}\right)$ with $68 \%$ and $90 \%$ quantiles for events that satisfy the containment selection.

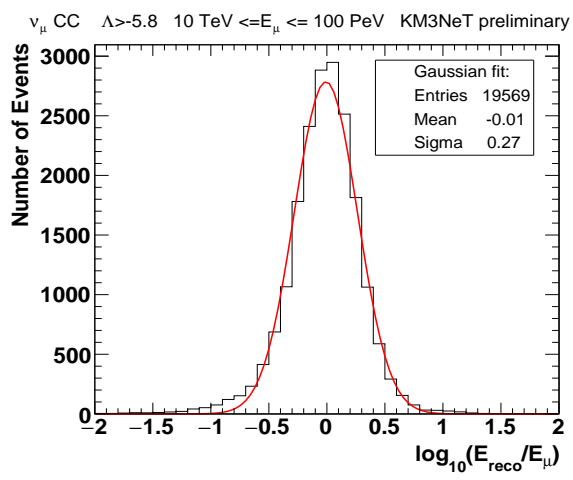

Figure 4: Distribution of $\log _{10}\left(E_{\text {reco }} / E_{\mu}\right)$ for events with $E_{\mu} \geq 10 \mathrm{TeV}$ that satisfy the containment selection with a Gaussian fit.

ii. The number of hit PMTs in the track direction reconstruction, $N_{\mathrm{PMT}}$, weighted according to the vertical distance from the track.

iii. The ratio of $N_{\mathrm{PMT}}$ over the number of PMTs that have been hit by direct Cherenkov photons for a given track geometry and PMT direction but have not recorded a signal.

iv. The total ToT of all PMTs (as a measure of charge) used in the track direction reconstruction.

\subsection{Results on Muon and Neutrino Energy Reconstruction}

The sample of CC $v_{\mu}$ simulated events is split in two different subsamples to avoid biases on the results. The first event subsample is used to train and test the Neural Network (NN) while the second subsample is used to evaluate the network performance. Once the weights of the interneuron connections of the NN have been determined, the second subsample of $v_{\mu}$ events is used for the estimation of the muon energy.

As a measure of the quality of this energy estimator, the median of $\log _{10}\left(E_{\mathrm{reco}} / E_{\mu}\right)$ is used, where $E_{\text {reco }}$ is the reconstructed muon energy and $E_{\mu}$ is the MC muon energy in the vicinity of the detector. This median is reported in Fig. 3 with $68 \%$ and $90 \%$ quantiles for events that satisfy the containment selection. A very good energy reconstruction is obtained for muons with $E_{\mu}>10 \mathrm{TeV}$ while for muon energies $E_{\mu}>1 \mathrm{PeV}$ the calculation suffers from limited statistics. The distribution of $\log _{10}\left(E_{\text {reco }} / E_{\mu}\right)$ for events that satisfy the containment selection is shown in Fig. 4. The energy resolution corresponds to $\simeq 0.27$ units in $\log _{10} E_{\mu}$ for $10 \mathrm{TeV} \leq E_{\mu} \leq 100 \mathrm{PeV}$. The efficiency of this energy estimator is investigated for all reconstructed muons with $\Lambda>-5.8$ (see section 3 ) that have survived the containment selection and is shown in Fig. 5.

The muon energy has been also reconstructed for all the events with a reconstructed direction and a cut $\Lambda>-5.8$ if they do not satisfy the containment selection. Muons failing the containment selection deposit only a small fraction of their energy inside the instrumented volume. In this case the muon energy cannot be reliably reconstructed and only a lower limit of the muon energy is calculated. The energy resolution reached for all events with $\Lambda>-5.8$ corresponds to $\simeq 0.28$ units in $\log _{10} E_{\mu}$ for $10 \mathrm{TeV} \leq E_{\mu} \leq 100 \mathrm{PeV}$. 


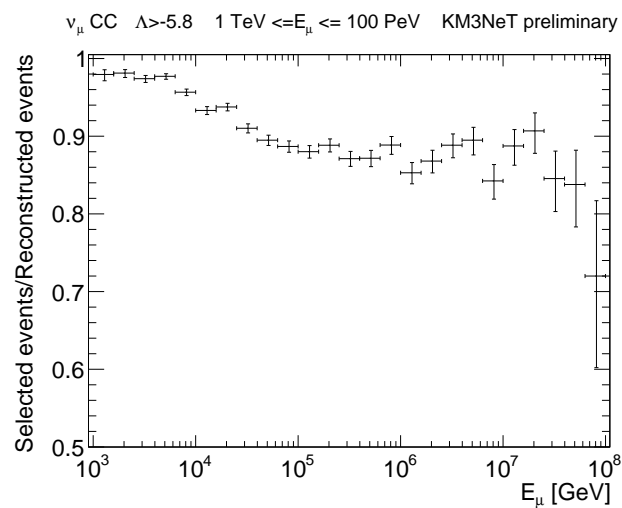

Figure 5: Fraction of the reconstructed events with $\Lambda>-5.8$ that survived the containment selection as function of the muon energy.

\section{Conclusions}

The algorithms described to reconstruct the muon direction and energy of events recorded by the KM3NeT/ARCA detector reaches a median angular resolution of $\sim 0.2^{\circ}$ and an energy resolution of $\sim 0.28$ in $\log \left(\mathrm{E}_{\mu}\right)$ for events with $\mathrm{E}_{v}$ above $10 \mathrm{TeV}$.

The performance of these reconstruction algorithms has also been tested for a detector configuration with a larger average horizontal distance between strings, i.e. $120 \mathrm{~m}$ instead of $90 \mathrm{~m}$ corresponding to a volume of $\sim 0.8 \mathrm{~km}^{3}$ per block. To estimate the muon energy the containment selection is appropriately adjusted in this case and a different Neural Network is trained. For events with $10 \mathrm{TeV} \leq E_{\mu} \leq 100 \mathrm{PeV}$ that satisfy the containment selection an energy resolution of $\simeq 0.25$ in $\log _{10} E_{\mu}$ was achieved, while for all events the energy resolution is $\simeq 0.26$. The reached median angular resolution is also almost the same w.r.t. the detector with $90 \mathrm{~m}$ spacing.

\section{References}

[1] A. Trovato et al. (KM3NeT Collaboration), these proceedings (ID=1113).

[2] M. G. Aartsen et al. (IceCube Collaboration), Phys. Rev. Lett. 113 (2014) 101101.

[3] http://www.km3net.org

[4] P. Piattelli et al. (KM3NeT Collaboration), these proceedings ( $I D=1158)$.

[5] B. Juergen et al. (KM3NeT Collaboration), these proceedings (ID=1140).

[6] A. Margiotta et al. (ANTARES Collaboration), NIM A 725 (2013) 98.

[7] P. Antonioli, et al. Astropart. Phys. 7 (1997) 357.

[8] Y. Becherini et al., Astropart. Phys. 25 (2006) 1.

[9] G. Riccobene et al., Astropart. Phys. 27, (2006) 1.

[10] S. Adrían-Martínez et al. (KM3NeT Collaboration), Eur. Phys. J. C74 (2014) 3056.

[11] M. G. Pellegriti et al. (KM3NeT Collaboration) AIP Conf. Proc. 1630 (2014) 94.

[12] A. J. Heijboer, PhD thesis, Universiteit van Amsterdam (2004).

[13] A. Hoecker et al., TMVAUsersGuide, (2013). 